\title{
Transfer effects of a cognitive strategy training for stroke patients with apraxia
}

Citation for published version (APA):

Geusgens, C. A., van Heugten, C. M., Cooijmans, J., Jolles, J., \& van Heuvel, W. J. A. (2007). Transfer effects of a cognitive strategy training for stroke patients with apraxia. Journal of Clinical and Experimental Neuropsychology, 29(8), 831-841. https://doi.org/10.1080/13803390601125971

Document status and date:

Published: 01/01/2007

DOI:

10.1080/13803390601125971

Document Version:

Publisher's PDF, also known as Version of record

\section{Please check the document version of this publication:}

- A submitted manuscript is the version of the article upon submission and before peer-review. There can be important differences between the submitted version and the official published version of record.

People interested in the research are advised to contact the author for the final version of the publication, or visit the DOI to the publisher's website.

- The final author version and the galley proof are versions of the publication after peer review.

- The final published version features the final layout of the paper including the volume, issue and page numbers.

Link to publication

\footnotetext{
General rights rights.

- You may freely distribute the URL identifying the publication in the public portal. please follow below link for the End User Agreement:

www.umlib.nl/taverne-license

Take down policy

If you believe that this document breaches copyright please contact us at:

repository@maastrichtuniversity.nl

providing details and we will investigate your claim.
}

Copyright and moral rights for the publications made accessible in the public portal are retained by the authors and/or other copyright owners and it is a condition of accessing publications that users recognise and abide by the legal requirements associated with these

- Users may download and print one copy of any publication from the public portal for the purpose of private study or research.

- You may not further distribute the material or use it for any profit-making activity or commercial gain

If the publication is distributed under the terms of Article $25 \mathrm{fa}$ of the Dutch Copyright Act, indicated by the "Taverne" license above, 


\title{
Transfer effects of a cognitive strategy training for stroke patients with apraxia
}

\author{
C. A. V. Geusgens, ${ }^{1,2}$ C. M. van Heugten, ${ }^{1,2}$ J. P. J. Cooijmans, ${ }^{1}$ J. Jolles, ${ }^{2,3}$ and \\ W. J. A. van den Heuvel ${ }^{1,4}$ \\ ${ }^{1}$ Institute for Rehabilitation Research, Hoensbroek, The Netherlands \\ ${ }^{2}$ Brain and Behavior Institute, Maastricht University, Maastricht, The Netherlands \\ ${ }^{3}$ Department of Psychiatry \& Neuropsychology, Maastricht University, Maastricht, \\ The Netherlands \\ ${ }^{4}$ Netherlands School of Primary Care Research (CaRe), Maastricht University, Maastricht, \\ The Netherlands
}

\begin{abstract}
The objective of this study was to evaluate transfer effects of cognitive strategy training for stroke patients with apraxia. During 8 weeks, 29 apraxic patients received cognitive strategy training to teach them how to perform activities of daily living (ADL) as independently as possible. ADL functioning was assessed at the rehabilitation centre at baseline and after 8 weeks of training. In addition, assessment took place at the patients' own homes after 8 weeks of training and 5 months after the start of the training. The performance of both trained and nontrained tasks was observed. Patients performed trained tasks and nontrained tasks at the same level of independency at the rehabilitation centre as well as at home, indicating transfer of training effects. These effects turned out to be stable over time.
\end{abstract}

\section{INTRODUCTION}

Apraxia is one of the more common cognitive impairments that can occur after stroke. It is an impairment affecting the purposeful execution of learned and meaningful skills that cannot be explained by primary motor or sensory impairments, nor by deficits in motivation, memory or comprehension (De Renzi, 1989). Like aphasia, apraxia usually occurs following left-hemisphere lesions (Basso, Capitani, Della Sala, Laiacona, \& Spinnler, 1987a). Although the exact prevalence of apraxia is not known, several studies reported apraxia to occur in 30 to $50 \%$ of all left-sided stroke patients (De Renzi, 1989; Donkervoort,
Dekker, van den Ende, Stehmann-Saris, \& Deelman, 2000; Zwinkels, Geusgens, van de Sande, \& van Heugten, 2004).

Apraxia can have a disabling effect on the performance of activities of daily living (ADL), causing a negative impact on everyday life. Therefore apraxia should be addressed during a rehabilitation treatment (Donkervoort, Dekker, Stehmann-Saris, \& Deelman, 2001; Goldenberg \& Hagmann, 1998; van Heugten, 2001a).

Van Heugten et al. (1998) developed a standardized occupational therapy program for lefthemisphere stroke patients with apraxia, which is based on teaching patients strategies to compensate for the presence of apraxia. The aim of the

Supported by The Netherlands Organization of Health Research and Development, Rehabilitation Research Program (Grant No. 14350009). The authors thank all participants and their families, as well as the rehabilitation teams of the healthcare institutions that participated in this study: the rehabilitation centres Amsterdam (Amsterdam), Het Roessingh (Enschede), Heliomare (Wijk aan Zee), De Hoogstraat (Utrecht), Rijndam (Rotterdam), Leijpark (Tilburg), Sophia (Den Haag), Blixembosch (Eindhoven), Sint Maartenskliniek (Nijmegen), De Trappenberg (Huizen), Rijnlands Revalidatiecentrum (Leiden), De Kastanjehof (Zutphen), Stichting Antaris (Amsterdam), and Stichting Vivre (Maastricht).

Address correspondence to Chantal Geusgens, Institute for Rehabilitation Research, PO Box 192, 6430 AD Hoensbroek, The Netherlands (E-mail: C.Geusgens@irv.nl). 
therapy is to maximize the patients' independence by improving ADL functioning; little change is expected in the severity of the apraxia itself. The effectiveness of this intervention program was shown in both a noncontrolled intervention study (van Heugten et al., 1998) and an experimental effect study (Donkervoort et al., 2001). Both studies showed that patients were able to function more independently on ADL tasks after receiving strategy training. However, in both studies ADL functioning was observed in the rehabilitation setting, whereas the aim of a rehabilitation program is to restore the patients' functioning to their greatest potential and maximum independence, hopefully resulting in independent functioning, the return to their own home, and participation in society.

For patients to function as independently as possible at home and in society, two types of transfer of treatment effects are needed. First, tasks that are taught in the rehabilitation setting should be transferred to the home setting. Second, transfer of intervention effects from trained tasks to nontrained (related) tasks is very important in terms of the clinical success of a therapy program, as therapists cannot possibly train all the difficulties and tasks that a patient will encounter after rehabilitation discharge.

In strategy training, the occurrence of transfer effects is expected as the training program is not aimed at relearning specific tasks, but at teaching patients new ways to handle the problems resulting from the impairment. However, a recently conducted review of literature revealed that relatively few studies on cognitive strategy training addressed the occurrence of transfer effects and that an even smaller part of these studies measured transfer in a standardized way (Geusgens, Winkens, van Heugten, Jolles, \& van den Heuvel, 2005). Moreover, to our knowledge, there are no studies that objectively compare the patient's functioning in the rehabilitation setting to the patient's functioning in his or her own home. However, in an exploratory study, indications were found for the occurrence of transfer of the apraxia training program from trained to nontrained tasks, although the study was not originally designed for the purpose of evaluating transfer. Patients significantly improved on nontrained tasks. This improvement was larger in the experimental group receiving strategy training than in the control group receiving apraxia treatment as usual, focusing mainly on (sensory)motor impairments (e.g., muscle tone, reflexes, controlled movements, muscle strength, contractures) and disability due to these impairments (Geusgens et al., 2006).
The goal of the present study was twofold. First, the occurrence of both transfer from the rehabilitation setting to the patient's home and transfer from trained to nontrained tasks was examined. For transfer effects to be demonstrated, it was hypothesized that nontrained tasks should be performed as independently as trained tasks, and that the performance at home should be as independent as the performance at the rehabilitation centre. Second, lasting transfer effects of the apraxia treatment program were examined at the patient's own home.

\section{METHOD}

\section{Patients}

Occupational therapists of the stroke teams of 16 Dutch healthcare institutions (14 rehabilitation centers and 2 rehabilitation teams of nursing homes) reported every patient that was referred to them and who complied with the inclusion criteria of this study. Patients were eligible if they met the following inclusion criteria: (a) a left-hemisphere stroke; (b) apraxia; and (c) the attending physician, the occupational therapist, and the patient judged the treatment of apraxia to be necessary. Exclusion criteria were: (a) age younger than 18 years; (b) time since stroke less than 4 weeks or more than 2 years; (c) a history of apraxia before the current stroke; (d) insufficient knowledge of the Dutch language (clinical judgement); (e) premorbid or present psychiatric or psychogeriatric pathologies; (f) a history of traumatic brain injury or brain tumor; (g) an alcohol or drug addiction; (h) a history of stroke causing a left-sided paresis that was present at the time of the study; (i) the patient did not finish primary school; (j) the patient did not agree to participate in the study.

The diagnosis of apraxia involved two steps. First, the patient's treating rehabilitation team made a clinical diagnosis. The patient was said to be apraxic if (a) the patient showed an inability (or restriction in the ability) to carry out purposeful tasks, and (b) this inability was not the result of a primary motor or sensory impairment, or deficit of comprehension, memory, or motivation. This diagnosis was based upon clinical judgment. There were no prescribed standardized observations or tests that the rehabilitation team had to use to come to this decision. Second, before final inclusion in the study, patients were tested by a trained neuropsychologist for severity of apraxia (van Heugten, Dekker, Deelman, Stehmann-Saris, \& Kinebanian, 1999b). Patients who showed no or 
minimal apraxic symptoms on the apraxia test (score above 85) were excluded from the study.

All patients and their families received verbal and written information about the study and gave verbal as well as written consent to participate.

\section{Study design}

Immediately after inclusion, each patient selected 6 ADL tasks from a list containing 14 tasks that had the same level of difficulty according to the classification of tasks of the Assessment of Motor and Process Skills (AMPS), in terms of both motor and process skill abilities needed to perform these tasks (Fisher, 1997; see Table 1). Patients were asked to select tasks that were relevant to them and that

TABLE 1

ADL tasks and number of participants that selected the tasks as trained or nontrained tasks

\begin{tabular}{|c|c|c|c|}
\hline Task & Trained & Nontrained & Total \\
\hline $\begin{array}{l}\text { Preparing a } \\
\text { cup of coffee/tea }\end{array}$ & 17 & 6 & 23 \\
\hline $\begin{array}{l}\text { Laying the table } \\
\text { for } 2 \text { persons }\end{array}$ & 13 & 4 & 17 \\
\hline Preparing bread & 9 & 6 & 15 \\
\hline $\begin{array}{l}\text { Dishwashing by } \\
\text { hand/loading and } \\
\text { starting } \\
\text { the dishwasher } \\
\text { ( } 5 \text { to } 8 \text { pieces) }\end{array}$ & 7 & 12 & 19 \\
\hline Brushing teeth & 7 & 7 & 14 \\
\hline Polishing shoes & 7 & 3 & 10 \\
\hline $\begin{array}{l}\text { Putting on a shirt, } \\
\text { sweatshirt, } \\
\text { or blouse }\end{array}$ & 7 & 3 & 10 \\
\hline $\begin{array}{l}\text { Folding laundry } \\
\text { (10 pieces) }\end{array}$ & 6 & 6 & 12 \\
\hline $\begin{array}{l}\text { Loading and starting } \\
\text { washing machine }\end{array}$ & 5 & 6 & 11 \\
\hline $\begin{array}{l}\text { Washing face } \\
\text { and upper body }\end{array}$ & 5 & 3 & 8 \\
\hline $\begin{array}{l}\text { Sweeping/mopping/ } \\
\text { vacuum cleaning }\end{array}$ & 4 & 8 & 12 \\
\hline $\begin{array}{l}\text { Watering } \\
2 \text { to } 3 \text { plants }\end{array}$ & 2 & 9 & 11 \\
\hline $\begin{array}{l}\text { Preparing a cup } \\
\text { of hot chocolate }\end{array}$ & 2 & 5 & 7 \\
\hline $\begin{array}{l}\text { Hand washing } \\
2 \text { pieces of laundry }\end{array}$ & 2 & 1 & 3 \\
\hline Other & 1 & 1 & 2 \\
\hline Total & 94 & 80 & 174 \\
\hline
\end{tabular}

Note. $\mathrm{ADL}=$ activities of daily living. After checking the registration of the content of the occupational therapy, not all tasks that should have been trained turned out to be trained during the 8 weeks of treatment. This table displays the number of participants in whom each task was observed, separated into the number of patients in whom the task was trained and in whom it was not trained during the first 8 weeks of the study period. they wanted to relearn. When needed, the occupational therapist and/or a significant other could help in selecting the tasks. The patient and the occupational therapist agreed to train 4 of the selected tasks, while the other 2 tasks would not be trained during the study period.

Prior to the start of the training, the performance on the six selected, standardized ADL tasks was observed at the clinic. A second measurement took place after an 8-week treatment period, in which four of the six selected tasks were trained. During this measurement, performance on the six ADL tasks was observed twice. Patients were asked to perform the tasks at the clinic, and, additionally, if the patient was living at home or if he or she went home for the weekend, observations of the six tasks took place at the patient's home as well. To minimize order effects, half the number of the patients was first observed at home, while the other half was first observed at the rehabilitation centre. A follow-up measurement took place 5 months after the start of the training. Patients were asked to perform the six ADL tasks at their own home. Treatment of patients during the follow-up period (Week 8 until Week 20) was not prescribed in specific study guidelines, except for the agreement not to train the two nontrained ADL tasks.

Measurements were conducted by an independent assessor (occupational therapist), who was trained in using all instruments that were applied in this study. The study protocol was approved by the ethics committees of all participating institutions.

\section{Intervention}

The intervention was a strategy training, specially designed for use by occupational therapists. The intervention was standardized by means of a treatment protocol, developed in an earlier study (van Heugten et al., 1998) Although the protocol was already implemented into Dutch occupational practice, the occupational therapists that participated in the study received an additional workshop to refresh their knowledge and insights into the use of the protocol.

At the rehabilitation centre, patients received 8 weeks of apraxia training. The frequency of training sessions was based on the clinical judgment of the treating occupational therapist and the rehabilitation team.

The intervention consisted of the standardized treatment program for left-hemisphere stroke patients with apraxia, developed by van Heugten and colleagues (van Heugten et al., 1998), which is based on teaching patients strategies to compensate 
for the presence of apraxia. By means of this program the patient is gradually taught more efficient strategies. The aim of the therapy is to maximize the patients' independence by improving ADL functioning. The specific problems of each individual patient are examined during a detailed diagnostic assessment of disability, consisting of standardized ADL observations. These problems will be the focus of attention during therapy; an intervention is chosen in correspondence with the observed problems. The treatment protocol is based on the framework of information processing. According to this framework, ADL tasks are conceptualized as being composed of three successive phases: The proper plan of action and the correct objects have to be selected (initiation and orientation phase), followed by adequate execution of the selected plan (execution phase), which has to be evaluated in terms of the result, and if necessary corrected (control and correction phase). Corresponding to these phases, the specific interventions focus on instructions, assistance, and feedback, respectively. Examples of the applied strategies include the use of verbalization and the use of pictures showing the correct order of task performance. This diagnostic assessment is strictly used for clinical purposes and is therefore independent of the study. For a more extensive description of the intervention and the strategies that can be used, we refer to the treatment protocol (Stehmann-Saris, 2005).

\section{Measurements}

\section{ADL functioning}

ADL functioning was measured in two ways:

1. ADL observations (van Heugten, Dekker, Deelman, Stehmann-Saris, \& Kinebanian, 1999a). These are a set of standardized observations, specially developed to assess disability in ADL functioning due to apraxia. The internal consistency and interobserver reliability of this observation procedure have been found to be good (van Heugten et al., 1999a; van Heugten, Dekker, Deelman, Stehmann-Saris, \& Kinebanian, 2000). Recently, the observations have been revised slightly, in order to make them even more consistent (Stehmann-Saris, 2005). The performance on six standardized tasks that were selected by the patient are observed. Each task was scored on four aspects: independence, initiation, execution, and control. While rating a task, the occupational therapist scores the help that he or she has to give to the patient to allow this patient to finish the task as independently as possible. Scores on the observations range from totally dependent (score of 0 ) to totally independent (score of 3).

2. Barthel ADL Index (Collin, Wade, Davies, \& Horne, 1988; Wade \& Collin, 1988). The Barthel Index of Tasks of Daily Living is a widely used, well-validated scale, which offers a simple and quick, clinically relevant method to identifying the most important physical disabilities. The score on the index ranges from 0 to 20 (Collin et al., 1988; Wade \& Collin, 1988).

\section{Apraxia}

The Apraxia Test is based on a test by De Renzi (De Renzi, 1989; van Heugten et al., 1999b). It consists of two subtests assessing (a) the ability to use objects as well as pantomime the use of objects (aimed at ideational apraxia), and (b) the ability to imitate gestures with the nonaffected ipsilateral arm (aimed at ideomotor apraxia). The maximum score of the total test is 90 . A score above 85 represents no or minimal apraxic symptoms. The internal consistency, the validity, and the interobserver reliability of the test are good (van Heugten et al., 1999b; Zwinkels et al., 2004). The apraxia test was included in this study as a measure to describe the severity of apraxia during the course of this study.

\section{Motor functioning}

The Functional Motor Test is a simplified version of the Action Research Arm Test (Lyle, 1981). The test measures the voluntary functional task of the arm and hand of the affected contralateral side. It consists of four items in which the patient has to use a type of grip (pinch, grip, or grasp) to pick up an object, move it forward, and put it down again. The maximum score on the test is 12 . The internal consistency is good (Donkervoort et al., 2001).

\section{Additional measures}

To monitor the patients' general cognitive functioning at baseline, standardized tests assessing verbal comprehension (Stichting Afasie Nederland Test, SAN test; maximum score is 45; Deelman, Liebrand, Koning-Haanstra, \& van de Burg, 1987), memory (Rivermead Behavioural Memory Test, RBMT; only nonverbally mediated items; maximum standard profile score is 16; Cockburn, Wilson, Baddeley, \& Hiorns, 1990; Wilson, Cockburn, \& Baddeley, 1985), and mental status (Cognitive Screening Test, CST, maximum score is 20; de Graaf \& Deelman, 1991) were used. 
Occupational therapists registered the amount and contents of the therapy sessions that patients received. In addition, information on demographic variables as well as stroke characteristics were registered.

\section{Statistical analyses}

For each patient, mean scores on the ADL observations for each of the six tasks separately were computed, based on the initiation, execution, and control score. This procedure is in accordance with the procedures that were used in earlier studies applying the ADL observations (Donkervoort, 2001; Geusgens et al., 2006; van Heugten et al., 1998). Next, for each patient a mean score for the performance on the six ADL tasks combined was computed, based on the mean score on the individual tasks. In addition, mean scores for ADL performance on trained and nontrained tasks, respectively, were computed for each patient, again based on the mean score on the individual tasks. These combined mean scores were used in the analyses and for computing effect sizes.

\section{Patient characteristics}

Descriptive statistics were used to present patient characteristics and test results at baseline. Independent-samples $t$ tests were used to evaluate whether patients who were lost to follow-up differed from patients who did complete the post treatment and follow-up observations. The following variables were compared between the two groups: age and baseline scores on the apraxia test, the ADL observations, the Barthel Index, motor functioning test, verbal comprehension test, and memory test.

\section{Improvement of functioning after 8 weeks of training}

A prerequisite for transfer effects to occur is the improvement of ADL functioning at the rehabilitation centre after treatment. A training program that does not have an effect on trained tasks in the training situation cannot be expected to transfer to other situations. In addition, if nontrained tasks do not improve after 8 weeks of training, transfer from trained to nontrained tasks could not have occurred. Therefore improvement of functioning at the rehabilitation centre after 8 weeks of training was assessed.

Paired-samples $t$ tests were used to evaluate improvements of ADL observations of trained tasks and nontrained tasks separately. In addition, a paired-samples $t$ test was used to evaluate improvements in the overall mean score of the ADL observations. In these comparisons the mean ADL score at baseline was not divided into trained and nontrained tasks, as none of the six observed tasks was trained at baseline and as there were no significant differences between tasks to be trained and nontrained tasks.

Paired-samples $t$ tests were also used to evaluate improvements of apraxia, motor functioning, and the Barthel Index. Based on the results of these comparisons, adjusted analyses were conducted for further evaluation of the treatment effects on the overall mean score of the ADL observations, as well as for the treatment effects on both trained and nontrained tasks separately. A general linear model (GLM) for repeated measures was used, in which we controlled for the changes in praxis. In addition, effect sizes were calculated by dividing the mean changes in scores by the standard deviation of the baseline score. An effect size of 0.2 is regarded as small, 0.5 as medium, and 0.8 or higher indicates a large effect (Cohen, 1988).

\section{Transfer of training effects after 8 weeks of training}

Two types of transfer were evaluated. First, transfer to new tasks was assessed by comparing performance on trained ADL tasks to that on nontrained ADL tasks, both at the rehabilitation centre and at home after 8 weeks of training. Second, transfer to a different situation was assessed after 8 weeks of training by comparing performance at the rehabilitation centre to performance at home for the mean scores on trained and nontrained tasks separately, as well as for the overall mean score on the ADL observations. To demonstrate transfer effects, no significant differences should be found between trained tasks and nontrained tasks or between the training situation and the home setting. These differences were assessed by using paired-samples $t$ tests. In addition, we performed post hoc power analyses, to infer beta, which represents the chance of a Type II error-that is, the chance that no statistically significant differences are found, although the means do differ.

\section{Lasting transfer effects at follow-up}

Paired-samples $t$ tests were used to evaluate lasting effects of the treatment program for the overall mean score on the ADL observations as well as for the mean score on trained and nontrained tasks separately, performed at home, 20 weeks after the start of the training. Lasting effects could be 
demonstrated if the performance at follow-up was as independent as was the performance directly after 8 weeks of training. Therefore, no significant differences should be found between the two observations. In addition, we performed post hoc power analyses, to infer beta.

In all analyses, the level of significance was set at .05 . Analyses were carried out using SPSS 11.0 for Windows. As most variables turned out not to follow a normal distribution, all analyses in which $t$ tests were used were repeated using the Wilcoxon Signed Rank test. Only parametric test results are reported in this paper, as conclusions based on the results of the parametric tests did not differ from the results of the nonparametric tests, and as the $t$ test is robust to moderate departures from normality (Hays, 1994).

\section{RESULTS}

\section{Patient characteristics}

Figure 1 shows the patient flow of the study. From May 2003 until December 2005 the occupational therapists reported 73 patients for participation in the study; 44 patients were excluded or lost to follow-up for various reasons.

Posttreatment observations took place both at the rehabilitation centre and at the patient's own home. A total of 22 patients were observed in both settings. A total of 3 patients were observed at home only, as they had already been discharged from the rehabilitation centre at the time of the observation; 4 patients were observed at the rehabilitation centre only, as they did not return home for the weekends yet. At follow-up, 19 patients were observed at their own home settings, whereas 5 patients were observed at the rehabilitation centre only, as they still had not been discharged from the centre. At baseline, a total of four tasks $(1.9 \%)$ could not be observed. In Week 8, six tasks (3.8\%) could not be observed in the rehabilitation centre, whereas the observations of eight tasks $(5.3 \%)$ are missing at the home setting. At follow-up, a total of seven tasks (6.1\%) could not be observed. All missing observations were due to planning problems in the rehabilitation centre or other practical problems. For participants who had missing observations, ADL mean scores were derived from the tasks that had been observed, thus using a smaller number of observations.

A group of 29 patients ( 22 men and 7 women) participated in this study. The mean age at inclusion was 60.5 years (standard deviation, $S D=9.1$; range 41-74), and the time postonset was 103.2 days $(S D=60.6$; range 36-285) Further patient characteristics and test results are displayed in Tables 2 and 3, respectively.

At baseline, participants who were lost to follow-up during the total study period of 20 weeks differed significantly in the score on the Barthel Index (lost: mean $=17.6, S D=2.7$; completed: mean $=14.74, S D=4.7), t(28)=2.15, p=.04$; and motor functioning (lost: mean $=10.7, S D=3.0$; completed: mean $=7.4, S D=4.4), t(27.5)=2.50$, $p=.02$. On both tests, participants who completed all observations scored significantly lower than participants who were lost to follow-up. There were no significant differences in age or in the scores on the apraxia test, the ADL observations, the Barthel Index, motor functioning, verbal comprehension, or memory. Participants who were lost to follow-up during the first 8 weeks of the study period did not differ on any of these variables.

\section{Improvement of functioning after 8 weeks of training}

During 8 weeks of training, the score on the ADL observations improved significantly in both trained and nontrained tasks. The overall mean score on the ADL observations improved as well (see Table 4). The effect sizes for the ADL observations indicate large effects, with values ranging from 0.9 to 1.0. The score on the apraxia test showed significant improvement as well. After controlling for this improvement all changes in ADL observations remained significant: overall, $F(1$, $24)=40.31, \quad p=.000 ;$ trained, $F(1,24)=25.46$, $p=.000$; nontrained, $F(1,24)=51.05, p=.000$.

\section{Transfer of training effects}

No significant differences were found between trained tasks and nontrained tasks, both at home and at the rehabilitation centre: home, $t(24)=0.20 ;$ rehabilitation centre, $t(25)=0.72$ (see Table 5). Also, no significant differences were found between functioning at the rehabilitation centre and that in the home setting, for the overall mean score in the ADL observations and for trained and nontrained tasks separately: overall, $t(21)=1.30$; trained tasks, $t(21)=0.81$; nontrained tasks, $t(21)=0.93$. Post hoc power analyses showed that beta was high, ranging from .76 to .94 . 


\section{Patients reported by the occupational therapists $(n=73)$}

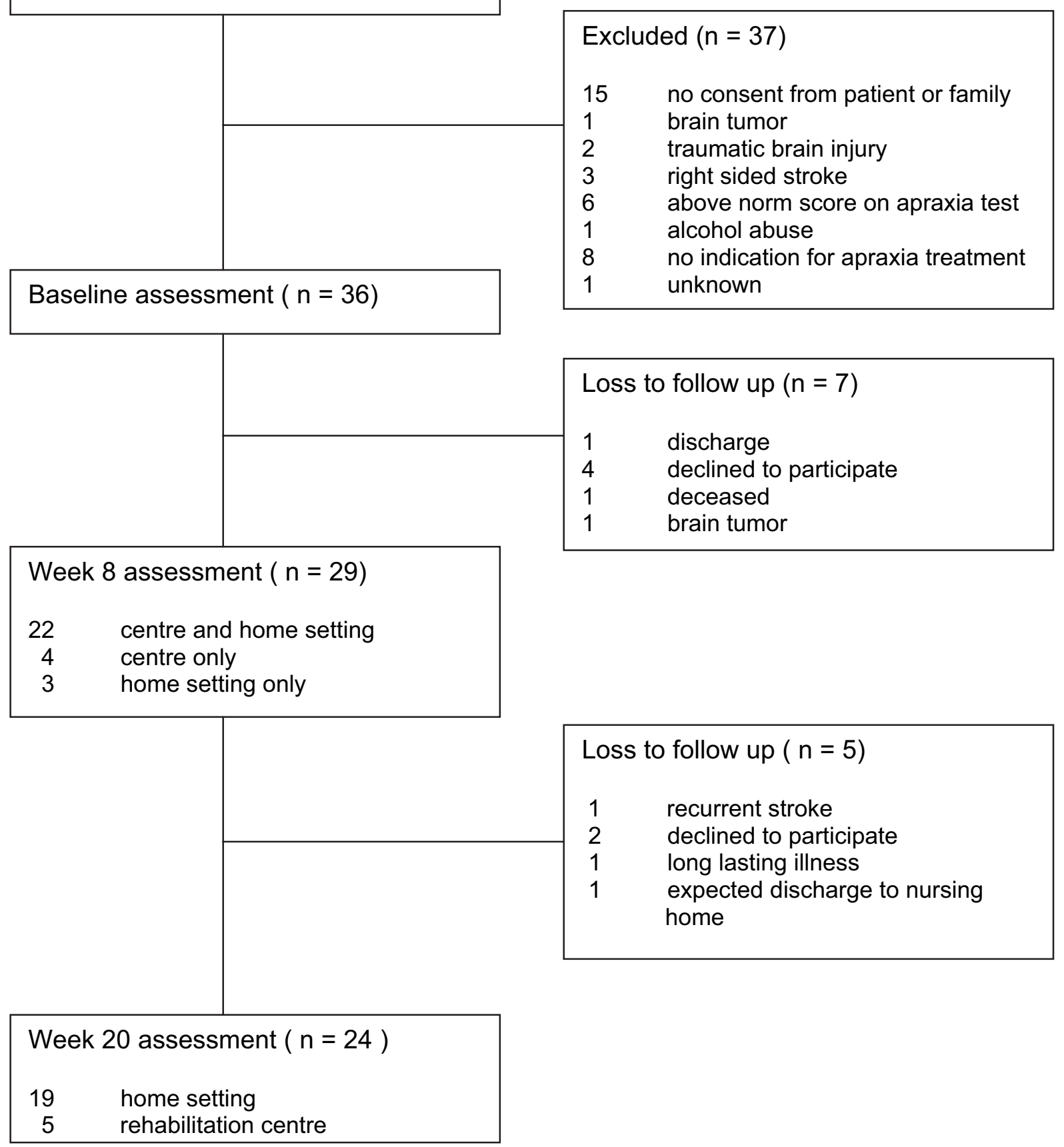

Figure 1. Flow chart of patients in the apraxia study.

\section{Lasting transfer effects at follow-up}

Performance on ADL observations at follow-up did not differ significantly from the performance directly after 8 weeks of training: overall, $t(17)=2.08$; trained tasks, $t(17)=1.78$; nontrained tasks, $t(17)=1.23$. Post hoc power analyses showed that beta was high, ranging from .50 to .79 . All observations taken into account took place at the patient's own home.

\section{DISCUSSION}

In this study, the transfer effect of cognitive strategy training for stroke patients with apraxia was evaluated. This is the first prospective larger scale study to examine this effect explicitly. By demonstrating the occurrence of transfer from trained to nontrained tasks, the results of a previous exploratory study can be confirmed. The exploratory 
TABLE 2

Demographic characteristics at baseline for patients who completed the observations after 8 weeks of training

\begin{tabular}{lrr}
\hline & $N$ & $\%$ \\
\hline Gender & & \\
Male & 22 & 75.9 \\
Female & 7 & 24.1 \\
Education & & \\
$\quad$ Low & & 13.8 \\
Middle & 4 & 55.2 \\
High & 16 & 13.8 \\
Unknown & 4 & 17.2 \\
Residence before stroke & 5 & \\
$\quad$ Living alone & & 17.2 \\
Living with partner & 5 & 75.9 \\
and/or children & 22 & 6.8 \\
Other & & 89.7 \\
Right-handedness & 2 & \\
Type of stroke & 26 & 31.0 \\
$\quad$ Hemorrhage & & 31.0 \\
Infarction & 9 & 70.0 \\
$\quad$ Unknown & 19 & \\
Recurrent stroke & 1 & \\
Hemiplegia & 9 & \\
\hline
\end{tabular}

Note. $N$ : number of participants.

${ }^{a}$ Education: low: primary school or less; middle: high school, vocational school, technical school; high: university. study demonstrated treatment effects of the same strategy training as that used in the present study (Geusgens et al., 2006). In addition, in the present study we also found indications for the occurrence of transfer from the rehabilitation setting to the home setting. Furthermore, we demonstrated the long-term effectiveness of the training program, for both trained and nontrained tasks.

In the present study, motor functioning remained stable over time during the 8 weeks of apraxia training, and although patients did improve significantly on the test for apraxia, the improvements on ADL observations in both trained and nontrained tasks remained significant after controlling for the improvement on the apraxia test. Moreover, the effect size for the apraxia test was small compared to the effect sizes for the ADL observations of both trained and nontrained ADL tasks. This finding supports the findings of other studies evaluating strategy training for stroke patients (Donkervoort et al., 2001; van Heugten et al., 1998), as well as the idea that the aim of strategy training is not to improve the impairment itself, but to teach patients new ways to handle problems resulting from an impairment (Ben-Yishay \& Diller, 1993).

At baseline, patients who were lost to follow-up during the total study period of 20 weeks scored

TABLE 3

Test results at baseline for patients who completed the observations after 8 weeks of training

\begin{tabular}{lcrrrr}
\hline & $N$ & Mean & $S D$ & Range & Max. \\
\hline Apraxia & 29 & 56.9 & 22.4 & $0-85$ & 90 \\
Functional Motor Test & 29 & 8.0 & 4.4 & $3-12$ & 12 \\
Barthel Index & 29 & 14.9 & 5.2 & $5-20$ & 20 \\
Verbal comprehension & 29 & 33.9 & 3.1 & $20-43$ & $4-14$ \\
RBMT standard profile score & $18^{\mathrm{a}}$ & 8.5 & 1.5 & $14-19$ & 16 \\
CST & $8^{\mathrm{a}}$ & 16.8 & 20 \\
\hline
\end{tabular}

Note. N: Number of participants. SD: standard deviation. Max.: maximum score. RBMT: Rivermead Behavioural Memory Test. CST: Cognitive Screening Test.

${ }^{a}$ Due to severe aphasia, these tests could not be administered to all participants.

TABLE 4

Improvement in functioning after 8 weeks of training

\begin{tabular}{lcccrrr}
\hline & Baseline $^{a}$ & Posttreatment $^{a}$ & $N$ & $t$ & $p$ & Effect size $^{b}$ \\
\hline ADL obs. total rehab. & $1.8(0.6)$ & $2.4(0.4)$ & 26 & 7.00 & .000 & 1.0 \\
ADL obs. trained rehab. & $1.8(0.6)$ & $2.4(0.4)$ & 26 & 5.48 & .000 & 1.0 \\
ADL obs. nontrained rehab. & $1.8(0.6)$ & $2.3(0.5)$ & 26 & 6.57 & .000 & 0.9 \\
Apraxia test & $56.9(22.4)$ & $67.3(19.6)$ & 29 & 3.99 & .000 & 0.5 \\
Functional Motor Test & $7.9(4.4)$ & $7.9(4.4)$ & 28 & -1.00 & .326 & -0.1 \\
Barthel Index & $14.8(5.2)$ & $16.8(4.1)$ & 28 & 3.70 & .001 & 0.4 \\
\hline
\end{tabular}

Note. $N$ : number of participants; ADL: activities of daily living; obs.: observations; rehab.: rehabilitation.

${ }^{a}$ Mean; standard deviation in parentheses. ${ }^{b}$ Effect sizes were calculated by dividing the mean changes in scores by the standard deviation of the baseline score. 
TABLE 5

Transfer effects from trained to nontrained tasks both at the rehabilitation centre and at home, after 8 weeks of training

\begin{tabular}{lllllll}
\hline & \multicolumn{2}{c}{ Tasks } & & & \\
\cline { 2 - 4 } & Trained $^{a}$ & Nontrained $^{a}$ & & $N$ & $t$ & $p$ \\
\hline ADL obs. rehab & $2.4(0.41)$ & $2.4(0.47)$ & 26 & 0.72 & .477 \\
ADL obs. home & $2.6(0.39)$ & $2.6(0.52)$ & 25 & 0.20 & .844 \\
\hline
\end{tabular}

Note. $N$ : number of participants. ADL: activities of daily living; obs. : observations; rehab.: rehabilitation centre.

${ }^{a}$ Mean; standard deviation in parentheses.

significantly higher on motor functioning and on the Barthel Index than did participants that completed all observations. Thus it can be concluded that the more severely impaired patients completed the study and demonstrated lasting transfer effects.

The occurrence of transfer is of great importance in terms of the clinical success of an intervention, as it reflects the aim of a rehabilitation program, which is for patients to function as independently as possible in their own environment and in society. Our findings show that after training, patients are indeed able to perform trained tasks and nontrained tasks at the same level of independency at the rehabilitation centre as well as at home. This finding could be explained by the fact that patients were able to apply the learned strategies in another situation (i.e., the home setting). Another possible explanation might be that old routines are triggered by the familiar home situation, making it possible for the patient to function independently without the use of a strategy (De Renzi, 1989).

Relatively few studies on cognitive strategy training address the occurrence of transfer, and an even smaller number of studies measure transfer in a standardized way (Geusgens et al., 2005). To our knowledge, this is the first study that objectively compared patients' functioning in the rehabilitation setting to their functioning in their own homes. Two other studies evaluated transfer of therapeutic effects of apraxia training from trained to nontrained tasks (Goldenberg, Daumuller, \& Hagmann, 2001; Goldenberg \& Hagmann, 1998). In both studies, no transfer was found as the training effects were shown to be restricted to the trained tasks. However, the training methods that were evaluated in these two studies are not comparable to the apraxia training that we used. In the first study, task-specific training was combined with a strategy training aimed at handling only one of the problems in ADL functioning that can occur in patients with apraxia (Goldenberg \& Hagmann, 1998), whereas in our training program for each individual patient a strategy is selected matching his or her specific problems. In the second study, task-specific training was combined with a strategy training that turned out to have no effect on trained tasks (Goldenberg et al., 2001). The differences between the intervention programs were discussed extensively in one of our previous papers (Geusgens et al., 2006).

Clinical relevance of these results is reflected by the fact that after 8 weeks of training, the majority of participants needed less help while performing both trained and nontrained ADL tasks at the rehabilitation centre as well as at home, indicating a higher level of independent functioning of the patient. Overall change on the independence score of the ADL observations was registered for each individual patient. Improvement on the independence score represents a reduction of help or care that patients need, in order to be able to perform the ADL tasks. Inspection of these independence scores showed that directly after 8 weeks of training, more than $60 \%$ of the participants showed an overall improvement on both trained and nontrained tasks, as well as in both situations, indicating that patients needed less help in the ADL task performance.

Spontaneous recovery of both apraxia and ADL functioning is important to consider when interpreting our findings. It is sometimes believed that apraxia recovers spontaneously (Basso et al., 1987b). However, other studies have shown apraxia to be persistent (Donkervoort, 2001; Poeck, 1985). The latter point of view was further confirmed by the data of Donkervoort et al. (2001) in which apraxia was observed to be enduring. Spontaneous recovery of ADL functions in patients with apraxia has not been investigated as thoroughly as the recovery of apraxia itself. There is, to our knowledge, only one study that looked into this matter. In this study spontaneous recovery of ADL functions did not occur (Goldenberg \& Hagmann, 1998). In addition, in the present study the time poststroke was more than 3 months on average, which exceeds the acute phase of recovery.

Our study demonstrates that after training, patients perform trained tasks and nontrained tasks at the same level of independency. Both the trained and nontrained tasks were of interest to the patients, as at the start of the study, patients were asked to select six tasks that were relevant for them to relearn. Therefore, patients could have been motivated to train themselves to perform the nontrained tasks when they were at home. We have not been able to control for this effect that could occur during home visits for the weekend or after 
discharge from the rehabilitation centre. However, this reflects the normal course of a rehabilitation process in which patients will return to their own environment, where they will have to learn to function as independently as possible. After discharge, patients will encounter tasks and situations that have not been trained during the rehabilitation process. Learning how to manage these tasks and situations represents the transfer of strategies that were trained at the rehabilitation centre.

Some methodological comments can be made concerning our study. First, at baseline, we did not observe ADL functioning at home. Including such a measurement would have added valuable information. As for now, no indication can be given of improvements in ADL functioning at home. However, we were not able to arrange such a measurement as patients could not go home in this phase of the rehabilitation process. Second, this study was not designed as a randomized clinical trial (RCT). However, conducting an RCT in which one group would have been subjected to a control treatment was difficult to arrange, as the use of the apraxia protocol was implemented into clinical occupational therapy (OT) practice in the Netherlands. Even if a protocol would have been used for performing the control treatment, occupational therapists would have been knowledgeable of the strategy training, making it very hard to create two independent treatment groups. In addition the RCT that was conducted previously already showed the effectiveness of the strategy training in comparison with a control treatment (Donkervoort et al., 2001). Based on this finding, the strategy training was published as a guideline for stroke rehabilitation in the Netherlands (van Heugten, 2001b). Third, our results should be interpreted with care, as post hoc power analyses showed that beta was large in our analyses investigating transfer effects. This suggests that differences between the ADL performances might exist although they were not demonstrated in the analyses. Factors that influence the value of beta are sample size, standard deviation, and the level of significance that was set for the analyses (alpha). In our study, the sample size was small, and the standard deviation was relatively large, compared to the range in scores of the ADL observations. However, beta represents the chance that the two means are not exactly the same, while in clinical practice, two observations will probably never be exactly the same. Therefore, it could be useful to choose an acceptable difference. Any difference smaller than this "acceptable difference" would be considered a meaningless difference, and the two observations would still be considered equal (Wellek, 2003).
Conducting post hoc power analyses based on this acceptable difference would probably provide a more appropriate beta. However, choosing an acceptable difference is difficult, considering the small range of scores of the ADL observations and considering the fact that there are no standards for choosing this difference, making it subjective. Fourth, a considerable number of patients in this study were lost to follow-up. However it was decided to refrain from inputting missing data, to make sure that the analyses were performed using the actual data. There is no indication of selective loss of data, because missing observations were due to practical and therefore individual reasons.

Despite these remarks, we feel that we have been able to provide more insight in the transfer of training effects of a cognitive strategy training for stroke patients with apraxia, as well as in the clinical relevance of this training program. However, more research is necessary to evaluate transfer effects of cognitive rehabilitation treatment and rehabilitation programs in general, as rehabilitation aims to teach patients ways to functioning as independently as possible, resulting in the return to their own home and participation in society. To reach this aim, the occurrence of transfer of training effects is a requirement, as most rehabilitation treatment is not administered at the patient's own home.

Original manuscript received 27 September 2006 Revised manuscript accepted 13 November 2006 First published online 24 December 2006

\section{REFERENCES}

Basso, A., Capitani, E., Della Sala, S., Laiacona, M., \& Spinnler, H. (1987a). Ideomotor apraxia: A study of initial severity. Acta Neurologica Scandinavica, 76, 142-146.

Basso, A., Capitani, E., Della Sala, S., Laiacona, M., \& Spinnler, H. (1987b). Recovery from ideomotor apraxia. A study on acute stroke patients. Brain, 110, 747-760.

Ben-Yishay, Y., \& Diller, L. (1993). Cognitive remediation in traumatic brain injury: Update and issues. Archives of Physical Medicine and Rehabilitation, 74, 204-213.

Cockburn, J., Wilson, B., Baddeley, A., \& Hiorns, R. (1990). Assessing everyday memory in patients with dysphasia. British Journal of Clinical Psychology, 29, 353-360.

Cohen, J. (1988). Statistical power analysis for the behavioral sciences. Hillsdale, NJ: Lawrence Erlbaum Associates, Inc.

Collin, C., Wade, D. T., Davies, S., \& Horne, V. (1988). The Barthel ADL Index: A reliability study. International Disability Studies, 10, 61-63.

Deelman, B. G., Liebrand, W. G. B., Koning-Haanstra, M., \& van de Burg, W. (1987). De SAN test: Een afasi- 
etest voor auditief en mondeling taalgebruik: Handleiding. Lisse, The Netherlands: Swets \& Zeitlinger.

de Graaf, A., \& Deelman, B. G. (1991). De cognitieve screeningtest: Handleiding. Lisse, The Netherlands: Swets \& Zeitlinger.

De Renzi, E. (1989). Apraxia. In J. G. F. Boller (Ed.), Handbook of neuropsychology (Vol. 2, pp. 245-263). Amsterdam: Elseviers Science Publishers.

Donkervoort, M. (2001). Apraxia following left hemisphere stroke: Prevalence, assessment and rehabilitation. Utrecht, The Netherlands: Vrije Universiteit.

Donkervoort, M., Dekker, J., Stehmann-Saris, J. C., \& Deelman, B. G. (2001). Efficacy of strategy training in left hemisphere stroke patients with apraxia: A randomized clinical trail. Neuropsychological Rehabilitation, 11, 549-566.

Donkervoort, M., Dekker, J., van den Ende, E., Stehmann-Saris, J. C., \& Deelman, B. G. (2000). Prevalence of apraxia among patients with a first left hemisphere stroke in rehabilitation centres and nursing homes. Clinical Rehabilitation, 14, 130-136.

Fisher, A. G. (1997). Assessment of motor and process skills: Manual (2nd ed.). Fort Collins, CO: Three Star Press.

Geusgens, C., van Heugten, C., Donkervoort, M., van den Ende, E., Jolles, J., \& van den Heuvel, W. (2006). Transfer of training effects in stroke patients with apraxia: An exploratory study. Neuropsychological Rehabilitation, 16, 213-229.

Geusgens, C. A. V., Winkens, I., van Heugten, C. M., Jolles, J., \& van den Heuvel, W. J. A. (2005). The occurrence and measurement of transfer in cognitive rehabilitation: A critical review. Manuscript submitted for publication.

Goldenberg, G., Daumuller, M., \& Hagmann, S. (2001). Assessment and therapy of complex activities of daily living in apraxia. Neuropsychological Rehabilitation, $11,147-169$.

Goldenberg, G., \& Hagmann, S. (1998). Therapy of activities of daily living in patients with apraxia. Neuropsychological Rehabilitation, 8, 123-141.

Hays, W. L. (1994). Statistics (5th ed.). Fort Worth, TX: Harcourt Brace College Publishers.

Lyle, R. C. (1981). A performance test for assessment of upper limb function in physical rehabilitation treatment and research. International Journal of Rehabilitation Research, 4, 483-492.

Poeck, K. (1985). Clues to the nature of disruptions to limb praxis. In E. A. Roy (Ed.), Neuropsychological studies of apraxia and related disorders (pp. 99-109). New York: North-Holland.

Stehmann-Saris, J. C. (2005). Occupational therapy guideline for assessment and treatment of apraxia following left hemisphere stroke. Amsterdam: Hogeschool van Amsterdam.

van Heugten, C. M. (2001a). Rehabiltitation and management of apraxia after stroke. Reviews in Clinical Gerontology, 11, 177-184.

van Heugten, C. M. (2001b). Revalidatie na een beroerte: Richtlijnen en aanbevelingen voor zorgverleners [Rehabilitation after stroke: Guidelines and recommendations for clinical practice]. The Hague, The Netherlands: Dutch Heartfoundation.

van Heugten, C. M., Dekker, J., Deelman, B. G., Stehmann-Saris, J. C., \& Kinebanian, A. (1999a). Assessment of disabilities in stroke patients with apraxia: Internal consistency and inter-observer reliability. The Occupational Therapy Journal of Research, 19, 55-73.

van Heugten, C. M., Dekker, J., Deelman, B. G., Stehmann-Saris, J. C., \& Kinebanian, A. (1999b). A diagnostic test for apraxia in stroke patients: Internal consistency and diagnostic value. The Clinical Neuropsychologist, 13, 182-192.

van Heugten, C. M., Dekker, J., Deelman, B. G., Stehmann-Saris, J. C., \& Kinebanian, A. (2000). Rehabilitation of stroke patients with apraxia: The role of additional cognitive and motor impairments. Disability and Rehabilitation, 22, 547-554.

van Heugten, C. M., Dekker, J., Deelman, B. G., van Dijk, A. J., Stehmann-Saris, J. C., \& Kinebanian, A. (1998). Outcome of strategy training in stroke patients with apraxia: A phase II study. Clinical Rehabilitation, 12, 294-303.

Wade, D. T., \& Collin, C. (1988). The Barthel ADL Index: A standard measure of physical disability? International Disability Studies, 10, 64-67.

Wellek, S. (2003). Testing statistical hypotheses of equivalence. Boca Raton, FL: Chapman \& Hall/CRC Press LLC.

Wilson, B., Cockburn, J., \& Baddeley, A. (1985). The Rivermead Behavioral Memory Test. Titchfield, UK: Thames Valley Test Co.

Zwinkels, A., Geusgens, C., van de Sande, P., \& van Heugten, C. (2004). Assessment of apraxia: Interrater reliability of a new apraxia test, association between apraxia and other cognitive deficits and prevalence of apraxia in a rehabilitation setting. Clinical Rehabilitation, 18, 819-827. 